\title{
OMC, libre-échange et guerres de religion
}

Tancrède VOITURIEZ

45 bis avenue de la Belle Gabrielle,

94736 Nogent-sur-Marne Cedex.

Fax : +33143947311

<tancrede.voituriez@cirad.fr>

Les négociations commerciales à I'OMC menées dans le cadre du Programme de Doha pour le développement ont été suspendues fin juillet 2006 en raison de « divergences entre les principaux protagonistes ». Les Chefs de délégation, s'exprimant lors d'une réunion informelle du Comité des négociations le 24 juillet, « sont convenus avec le Directeur général de I'OMC, Pascal Lamy, qu'il s'agissait d'un revers pour tous les Membres». On ignore s'il s'agit d'une formule de politesse destinée à réduire la déception du directeur général et à le remercier de ses efforts, ou si véritablement chaque pays membre souhaitait obtenir un accord sans parvenir à offrir suffisamment à ses partenaires pour autant. Et sans préjuger des coups de théâtre qui restent encore possibles, il n'est pas inutile de revenir sur le lent enlisement du projet de libéralisation commerciale au service du développement dont l'OMC, avec le « cycle de Doha ", a été un enthousiaste promoteur.

L'ambition de mettre le commerce au service du développement est explicite depuis I'inauguration du cycle de négociation de I'OMC à Doha en 2001. Jeune organisation héritière du GATT, I'OMC garde pour mandat d'accroître la production et les échanges internationaux " par la réduction substantielle des tarifs douaniers et des autres obstacles au commerce, et par l'élimination des discriminations en matière d'échanges ». Le développement n'apparaît pas dans ses objectifs, si ce n'est en fin de phrase de son Préambule, où il est rappelé que I'usage optimal des ressources de la planète, auquel doit contribuer l'Organisation, doit être " conforme à l'objectif de développement durable", ce qui est à la fois grandiose et vague, et n'engage à rien. L'OMC à l'origine prolonge ainsi I'œuvre du GATT, levant discriminations et barrières aux échanges, prioritairement.

L'irruption du «développement " dans les négociations commerciales, que l'on aurait pu croire superficielle et bénigne, a bouleversé la

\begin{abstract}
We try to find out in this paper the very reasons why negotiating a trade liberalisation round for development within the WTO is so arduous. We elaborate on the expected impacts from liberalisation, and on the advertising often biased made about them by the free-trade clergymen
\end{abstract}

Key words: WTO, free trade, trade liberalisation

rhétorique et les argumentaires de négociation, plaçant les pays riches dans la position difficile d'être généreux pour les autres - pour le développement - et égoïstes pour euxmêmes sous peine d'encourir la colère des parlementaires et des groupes d'affaires nationaux. Les pays moins riches quant à eux (non $\mathrm{OCDE}$, pour faire court) ont très naturellement su se faire les porte-drapeaux de la cause du développement, réunissant sous une même bannière des pays aux revenus, aux institutions, et partant, aux objectifs de négociation raisonnablement différents. Dans ce grand théâtre, la négociation à l'arraché, entre pays riches, d'un accord ensuite imposé aux pays moins riches, comme ce fut le cas à Marrakech en 1994 lors du précédent cycle de négociation (cycle dit d'Uruguay, couvrant la période 19861994 au terme duquel naîtra l'OMC), n'était plus possible, et c'est bien pour avoir essayé de reproduire des négociations entre happy few que les États-Unis et l'Europe ont provoqué la création d'un groupe de pays émergents, le G20, qui n'aura de cesse de jauger à l'aune du développement la qualité des brouillons $d^{\prime}$ accord produits au fil des ans. Un premier problème est ainsi venu perturber le théâtre des délibérations, problème de procédure plus que de fond, lié à l'obligation de négocier un texte entre un nombre accru de pays aux structures commerciales et aux revenus différents.

Dans ce théâtre encore, on ne parle que de justice ; il fallait que l'accord soit juste, le commerce mondial tout autant; fair trade for all ou un commerce juste pour tous étant très symptomatiquement le titre choisi par un Nobel, Joseph Stiglitz, et son coauteur, Andrew Charlton pour leur ouvrage sorti durant les dernières semaines de 2005 en marge de la Conférence ministérielle de l'OMC à Hong-Kong. De quelle justice parle-t-on? Une justice de réparation tout d'abord, puisqu'avec le cycle de Doha, les pays membres de l'OMC se sont engagés à corriger des injustices passées, qui tant dans les modalités de négociation que dans le contenu et l'impact des accords, faisaient la part trop belle aux pays de l'OCDE. La libéralisation commerciale devait ensuite être juste selon le sens plus consensuel donné au « développement » par les agences d'aide et l'ONU, selon lequel « développement » et « réduction de la pauvreté » pouvaient être tenus pour des objectifs substituables I'un à l'autre. Une libéralisation juste devait ainsi accroitre prioritairement le sort des pays les moins riches, et à l'intérieur de ceux-ci, concourir à améliorer la situation des ménages les plus défavorisés. Accroître d'autant plus le PIB que celui-ci est faible; accroître les revenus des premiers « tranches » ou premiers déciles de population. Et ce sont bien là les deux autres problèmes qu'ont rencontrés les négociations.

Les économistes n'ont pas ménagé leurs efforts pour vérifier que pour chacune des différentes moutures des textes d'accord en discussion, les gains de la libéralisation commerciale avancés par leurs modèles satisfaisaient les ambitions de justice que l'on vient de décrire à grands traits. Les pays en développement doivent en être les premiers bénéficiaires. Au sein de ces pays, les pauvres doivent, grâce à la libéralisation, voir leur condition s'améliorer. Des modèles mondiaux, modèles mathématiques élaborés lors du précédent cycle (1986-1994) et raffinés pour l'occasion, ont souligné le coût du protectionnisme et des subventions pour les pays mêmes qui mettaient en place ces mesures. Pour se limiter à l'exemple agricole, des pays ou des régions telles que l'Union européenne, les États-Unis et le Japon, parce que les plus interventionnistes, se trouvaient ainsi parmi les premiers bénéficiaires de la libéralisation. Ce n'était pas exactement le "développement» ou la justice que l'on attendait. Dans le même temps, les estimations de la variation de la pauvreté induite par la libéralisation produisaient des chiffres positifs ou négatifs selon les pays, sans effet univoque dans un sens comme 
dans un autre, au point que l'on s'interroge sur l'opportunité d'avoir claironné si hâtivement les vertus enrichissantes, et systématiques, de l'ouverture commerciale une fois celle-ci mesurée à l'échelle des ménages.

Difficulté de négocier entre un nombre accru de pays à structures commerciales et revenus différents, mais à pouvoir égal ou presque en raison de l'impératif de développement impact positif discutable, sinon douteux, de la libéralisation sur les pays, et à l'intérieur des pays, sur les ménages les plus pauvres; tels sont les trois grands problèmes qui ont inexorablement enfoncé dans le sable les négociations. Un quatrième, plus dissimulé, a contribué à l'enlisement, qui est celui de l'impasse dans laquelle se sont fourvoyés les grands prêtres de la libéralisation.

\section{Le clergé du libre-échange}

Une illustration caricaturale peut être donnée par la publication en 2005 d'une étude de la Banque mondiale dirigée par Kym Anderson et Will Martin, étude qui conclut sans ambages que les gains de la libéralisation sont « énormes ", qu'ils «bénéficieront de manière disproportionnée aux pays en développement ", et $s^{\prime}$ obtiendront par une libéralisation prioritaire des subventions agricoles, et ce, au bénéfice probable des pauvres. Que du bonheur, donc, de quoi se plaint-on? Les conclusions sont remarquables pour deux raisons. La première est qu'elles sont malhonnêtes, puisqu'elles avancent des gains potentiels d'une libéralisation complète (autrement dit, le libre-échange) comme argument pour la libéralisation forcément partielle à laquelle aboutirait le cycle de Doha - on ne négocie pas le libre-échange à I'OMC, loin s'en faut. À titre d'analogie, c'est un peu comme si votre concessionnaire vous vendait un véhicule pour ses performances en matière de vitesse, performances dont il omettrait de vous signaler qu'elles sont été mesurées en laboratoire dans un monde sans frottement. La deuxième raison, renforçant la première, est que le détail des résultats démontre les ambiguïtés et les nuances que comporteraient les effets du Cycle sur les pays en développement (PED), et en particulier sur les pays les moins avancés. Or, ces ambiguïtés et nuances ont été omises en conclusion, tout comme dans la petite plaquette destinée à la presse. Tout ceci ne peut échapper au premier lecteur venu, ce qui rend encore plus étrange la brutalité, comme désespérée, de l'argumentation d'Anderson et Martin. Mark Weisbrot par exemple, patron du très sérieux Center for Economic and Policy Research (CEPR) soulignait dans un bulletin de presse le 22 novembre 2005 : « II est étrange de voir les gains potentiels du Doha Round

\section{Encadré 1}

\section{La réponse du CEPR à l'évaluation de l'impact de Doha par Anderson et Martin (2005)}

La suppression des droits de douane, des subventions et des soutiens internes devrait accroitre le bien-être global de près de 300 milliards de dollars par an à l'horizon 2015, selon une étude de la Banque mondiale intitulée Agricultural Trade Reform and the Doha Development Agenda (décembre 2005). Cette information est trompeuse selon le Center for Economic and Policy Research, pour les raisons suivantes:

1) La suppression des subventions agricoles à l'exportation et des programmes de soutien interne devrait en réalité entraîner une perte nette pour les pays en développement. Ceci s'explique principalement par la hausse attendue des prix des produits agricoles et alimentaires consécutivement à ces réformes.

2) Les quelque 300 milliards de dollars annoncés par la Banque mondiale (en réalité 287 milliards de dollars) représentent seulement 0,7 \% du PIB mondial en 2015. Pour prendre un exemple, comparez deux scénarios: le premier dans lequel le Cycle de Doha échoue sans qu'aucune libéralisation ne survienne, le second dans lequel tous les obstacles au commerce des marchandises sont éliminés. La différence entre les deux scénarios s'élève à $0,7 \%$ du PIB. Par exemple, un pays dont le revenu annuel serait de 1000 dollars par personne en 2015 sans libéralisation commerciale aurait un revenu de $1007 \$$ dans le cas d'une libéralisation complète du commerce de marchandises.

3) Les pays en développement considérés dans leur ensemble gagneraient 86 milliards de dollars, soit $0,8 \%$ du PIB en cas de libéralisation complète. Toutefois, environ la moitié de ces gains proviendrait de la libéralisation des propres politiques commerciales des pays en développement. Cela signifie que même si le Cycle de Doha devait échouer, la plupart des gains à la libéralisation seraient encore accessibles à ces pays puisque n'importe quel pays peut libéraliser ses importations à n'importe quel moment, sans règles I'obligeant à le faire.

4) Les 287 milliards de gains procèdent d'une libéralisation complète, qui n'est pas ce que l'on attend du Cycle de Doha. Les estimations de gains par la Banque dans des scénarios plus réalistes sont beaucoup plus faibles : entre 17,9 et 119,3 milliards, ou seulement 0,04 et 0,28 \% du PIB mondial. Encore une fois, la plupart de ces gains très modestes seraient toujours accessibles aux pays en développement même si Doha devait échouer.

5) Même un cycle de Doha conduit avec succès à son terme modifierait à peine l'incidence de la pauvreté : selon l'étude, le nombre de personnes vivant dans la pauvreté en 2016 serait réduit d'une proportion située entre 0,4 et $1 \%$ (soit entre 2,5 et 6,3 millions de personnes).

exagérés bien au-delà de ce que la recherche économique standard indique. » Et de conclure en suggérant que la Banque augmente ses efforts pour expliquer précisément les résultats de ses recherches sur les gains potentiels de la libéralisation (encadré 1).

Démontrer les gains pour les PED, et en particulier les plus pauvres d'entre eux, du Cycle de Doha est impossible si l'on se donne l'ambition de leur octroyer un caractère systématique. On le savait bien avant que l'étude d'Anderson et Martin ne paraisse ; tout cela a été confirmé depuis. Alors pourquoi publier pareilles énormités? Pourquoi diable certains économistes du département du commerce de la Banque mondiale se transforment-ils à l'occasion en prêtres et assènent-ils des prédictions qui relèvent de la croyance et de la religion?

On connaît une partie de la réponse : quand bien même il serait justifié (" efficace » selon leur terme) que l'État intervienne, les gains $d^{\prime}$ une intervention publique sont faibles comparativement au risque de voir les droits de douane et autres subventions dévoyées et utilisées à des fins personnelles et électorales, pour un résultat finalement négatif si on le compare au libre-échange. Certains arguments théoriques existent contre la libéralisation, tout cela est admis par les économistes même les plus libéraux (au sens français du terme), mais grosso modo, l'État étant ce qu'il est, mieux vaut ne pas compter sur lui et préférer se rapprocher au plus près du libre-échange. Et pour ne pas faire naître l'espoir que l'État peut accompagner efficacement le marché, espoir qu'il faudrait tout aussitôt doucher par des raisonnements de rabat-joie sur la difficulté pratique d'avoir un État efficace, tous les arguments sont bons, mêmes les plus fallacieux déguisés sous les atours des mathématiques et de la modélisation.

Anderson et Martin sont sincèrement convaincus que le libre-échange est la situation la plus favorable, en pratique, que peut connaître un pays. Cette situation peut créer des pauvres, être défavorable aux plus démunis, tout cela 
reste anecdotique à côté du spectre de l'immense gâchis qu'élève une intervention publique même animée à l'origine des meilleures intentions. D'une référence théorique permettant la comparaison des effets de différentes mesures d'intervention publique dans une économie, le libre-échange est devenu, chez Anderson et Martin, une référence normative de politique, de sorte que les plus furieux des altermondialistes ont pu s'en donner à cœur joie et dénoncer l'entreprise de propagande à laquelle contribuent, en toute conscience, les économistes « libéraux » dans leurs exercices de modélisation des échanges. À vouloir vendre la libéralisation pour ce qu'elle ne peut prétendre résoudre, le Département du commerce de la Banque mondiale a ainsi réussi l'exploit d'unir dans un même front l'ultragauche altermondialiste et des économistes modérés; imaginez en France Olivier Besancenot serrant la main de Dominique Strauss-Kahn ; personne n'y aurait pensé, la Banque mondiale I'a fait.

Maintenant que les négociations sont dans le sable, les deux questions qui se posent sont les suivantes. Si la libéralisation n'est pas le remède miracle qui réglera les problèmes de développement, quel rôle I'OMC peut-elle jouer pour améliorer le sort des pays les moins favorisés? Est-il même raisonnable de la mettre à contribution dans la définition et la mise en œuvre des politiques de développement ? On ne peut rendre compte ici de la diversité des réponses apportées à cette question, et s'il ne fallait en retenir qu'une seule, sans doute celle de Stiglitz et Charlton est-elle des plus éclairantes, pour le diagnostic et les solutions qu'elle suggère, mais aussi pour les divisions qu'elle souligne au sein du groupe, il est vrai très vaste, des organisations non gouvernementales prenant position sur les questions de commerce et de développement.

\section{Le front anti-clérical}

Réunion de plusieurs papiers commandités par le secrétariat du Commonwealth en 2004, I'ouvrage de Stiglitz et Charlton, Fair Trade for All, paru en décembre 2005 aux presses universitaires d'Oxford, propose quelques grandes lignes de réforme de I'OMC qui feraient du commerce un réel outil de développement. L'ambition de départ n'est donc pas de se défier du commerce international et de $s^{\prime} y$ soustraire mais bien de s'en servir comme levier de développement. L'image d'un Stiglitz pourfendeur des dérives de la Banque mondiale et du FMI en matière de doctrine macroéconomique, véhiculée par ses ouvrages grand public comme La Grande désillusion ou Quand le capitalisme perd la tête, ne peut être reproduite à I'identique en matière de commerce. Stiglitz n'est pas opposé à la libéralisation, ce qu'il condamne, avec Charlton, ce sont les règles et les principes qui en organisent le déroulement, règles et principes inscrits dans les textes de I'OMC qui arrangent surtout les grands pays, pour avoir été rédigé par ceux-ci. Ainsi, soulignent les auteurs, des secteurs intensifs en main-d'œuvre dans les pays en développement, comme l'agriculture et les textiles, que I'on pourrait supposer donc à l'avantage de ceux-ci, figurent dans l'agenda de négociation parmi les derniers à être soumis à la libéralisation. Dans le même temps, des accords très contraignants pour les $\mathrm{PED}$, portant sur la propriété intellectuelle, les normes sanitaires et phytosanitaires, les obstacles techniques au commerce, sans oublier le règlement des différends, ont été rédigés et mis en place à un rythme trépidant.

Un second élément de diagnostic présenté dans l'ouvrage porte sur les exagérations des modèles de simulation des effets de la libéralisation, auxquels deux critiques fondamentales sont apportées : la première est que ces modèles sont avant tout statiques, or le développement, comme le nom peut l'indiquer pourtant, est avant tout une affaire dynamique. Ils ne peuvent donc rien dire de bien sérieux sur les effets de la libéralisation au cours du temps. Cette ignorance n'est pas honteuse, elle est celle de la discipline économique, ajoutent les auteurs : aucun modèle validé par des données ne permet de prévoir aujourd'hui quel est l'effet de l'ouverture commercial sur le développement. Point. Les modèles existants, par ailleurs, pour l'écrasante majorité d'entre eux, considèrent que l'économie tourne à pleinemploi, et que la main invisible du marché déplace les travailleurs d'un secteur à un autre, où plus productifs (pour peu que le fonctionnement de l'économie soit « parfait»), ils verront leur rendement, et donc leur rémunération, augmenter. Enfin, même en raisonnement statique, la priorité mise dans la libéralisation commerciale des pays en développement n'est pas fondée. Supposez qu'une économie, imparfaite, c'est-à-dire souffrant selon le jargon de multiples «défaillances de marché " (chômage, restriction de crédit, absence d'assurance par exemple), produise 100 avec une structure de protection douanière donnée. Sans défaillance de marché, elle pourrait produire 200, avec la même structure de protection. Ce que disent les modèles, suggèrent Stiglitz et Charlton, c'est qu'en libéralisant, l'économie va passer d'une production de 200 à une production de 210. La réalité, c'est qu'en libéralisant, l'économie qui produisait 100 et non 200 (en raison des défaillances de marché non prises en compte par les modèles) n'a absolument aucune chance de produire 210. Et les auteurs d'ajouter, malicieux, qu'il se peut même qu'elle produise 55 , ou qu'elle continue de produire 60 , mais avec un petit peu plus de pauvres - tout cela les modèles n'en savent rien. Ce sont les conditions de fonctionnement des marchés, dans chaque pays, qui déterminent au cas par cas la réponse. La priorité n'est donc pas de libéraliser, mais de faire fonctionner correctement les marchés afin de passer d'une production de 100 à une production de 200 ; pour cela, l'État et I'intervention publique sont nécessaires; ensuite, oui, libéraliser permet d'ajouter un petit plus aux gains déjà engrangés.

La force du raisonnement de Stiglitz et Charlton est qu'il renvoie dos à dos le clergé freetrader et le clergé protectionniste. Les économies aux institutions de marché (crédit, assurance, propriété privée) à peu près efficaces sont proches d'une production de 200, pour conserver le même exemple. Elles ont donc intérêt à libéraliser, pour augmenter encore un peu la taille du gâteau produit par l'économie nationale. C'est le cas des pays de l'OCDE. Les pays aux institutions défaillantes ont $d$ 'abord intérêt de renforcer celles-ci avant de libéraliser leurs importations. C'est le cas des pays en développement, à tout le moins, des plus pauvres d'entre eux. Si le free trade ne peut être une recette pour tout le monde, le protectionnisme ne peut l'être non plus.

Les propositions principales de l'ouvrage sont doubles. La première vise à introduire une asymétrie entre pays dans l'obligation d'accroissement de l'accès au marché (l'ouverture commerciale, pour faire court) offert aux autres pays. L'accès aux marchés extérieurs, considéré comme un levier nécessaire du développement, doit être consenti intégralement par chaque pays membre de I'OMC à tout pays en développement plus petit que lui (la taille étant mesuré par le PIB) et plus pauvre (la richesse étant mesuré par le PIB par tête). En plaçant en abscisse la richesse et en ordonnée la taille, en positionnant ensuite par ces axes les différents pays, on obtient un nuage de pays dont ceux situés en haut à droite devront garantir à ceux situés sous eux un accès complet au marché (figure 1, tirée d'une présentation de Andrew Charlon à la Conférence ABDCE, Banque Mondiale, Tokyo, 21-22 mai 2006).

La seconde proposition vise à mettre plus volontairement à contribution l'aide publique au développement afin de «faire passer » les pays d'une production de 100 à une production de 200, dans notre exemple ; de préparer et d'accompagner l'intégration au marché mondial par le renforcement des institutions publiques domestiques.

Ces deux propositions, et notamment la première, n'ont pas fait l'unanimité, loin s'en faut, au premier motif qu'elles violent un des principes fondamentaux du CATT et de I'OMC, qui 


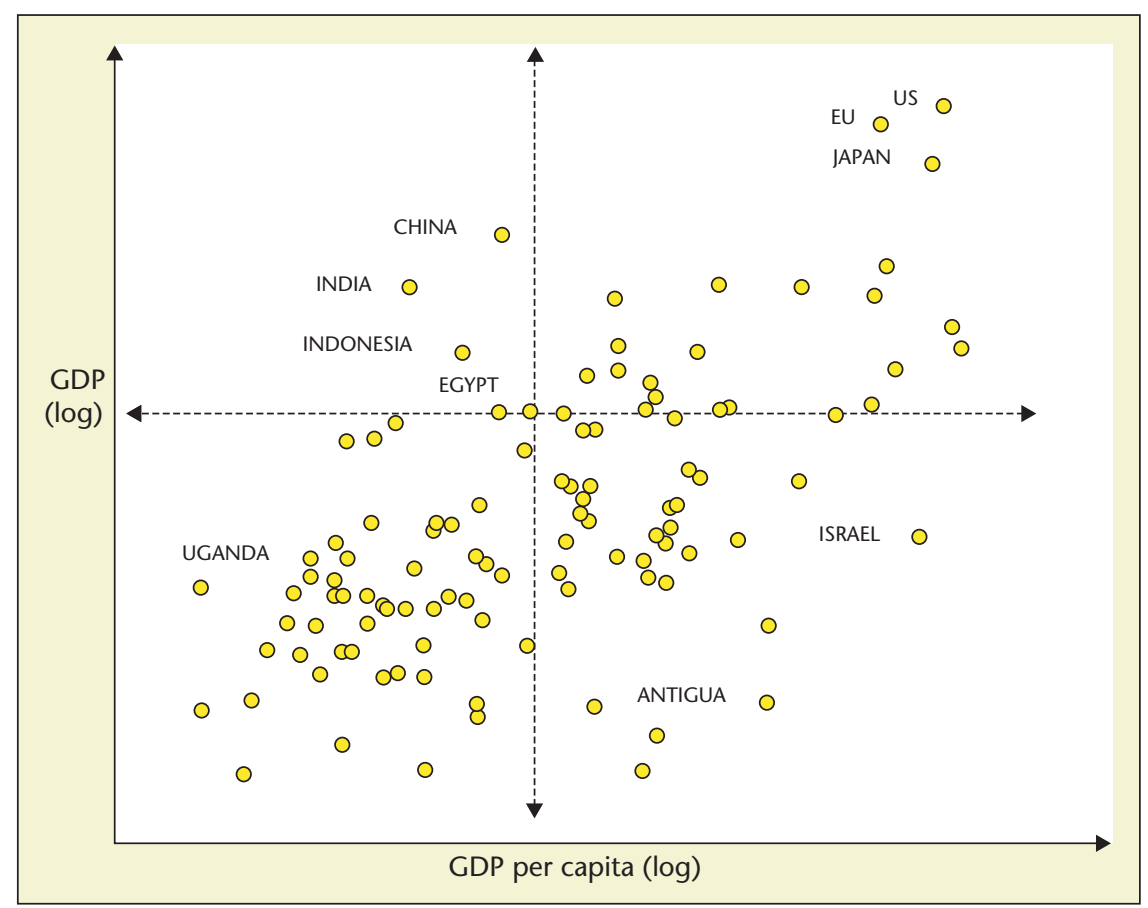

Figure 1. D'après une présentation de Andrew Charlon à la Conférence ABDCE, Banque mondiale, Tokyo, 21-22 mai 2006.

est celui de la réciprocité. À l'OMC un pays peut exiger à hauteur de ce qu'il offre, en terme d'accès au marché (baisse réciproque des droits de douane). Chez Stiglitz et Charlton, ce n'est plus le cas. Vous ouvrez intégralement à plus petit et plus pauvre que vous, ce qui oblige
I'Union européenne et les États-Unis par exemple à bouleverser la structure de leurs importations. Peu réalistes en l'état de léthargie actuelle des négociations, ces propositions présentent l'intérêt néanmoins de souligner, pour notre gouverne, qu'il n'y a pas de modèle agricole, ni de préférence communautaire et autre souveraineté alimentaire compatibles entre elles dans des pays à taille et à revenus différents. Cette précision faite, l'ajournement des négociations de libéralisation, laissant un Nord libre de protéger et subventionner et un Sud sans accès complet aux marchés extérieurs, est une situation d'injustice qu'il n'est pas idiot de voir l'OMC contribuer à renverser. Ambition raisonnable pour une organisation mêlant pays développés et pays en développement, et se préoccupant à peu près exclusivement de l'accès au marché de ses pays membres. Quant aux politiques de développement en tant que telles, elles restent l'obligation des gouvernements, avec le soutien des agences d'aide, et des banques de développement, raison pour laquelle, sans doute, certains économistes de la Banque mondiale, tout à leur phobie de la dépense publique, n'ont eu de cesse d'enjoliver les gains à l'échange, réduisant d'autant l'espace dévolu aux politiques, plus imaginatives et incertaines, qu'exige le développement. 